\title{
Omalizumab is effective in patients with chronic spontaneous urticaria plus multiple chronic inducible urticaria
}

${ }^{1}$ Division of Immune-mediated skin diseases, I.M. Sechenov First Moscow State Medical University (Sechenov University), Moscow, Russian Federation

${ }^{2}$ Department of Dermatology and Allergy, Charité - Universitätsmedizin Berlin, Berlin, Germany

\section{KEY WORDS}

Urticaria; omalizumab; treatment; chronic

spontaneous urticaria; chronic inducible

urticaria.

\author{
Corresponding author \\ Pavel Kolkhir \\ Allergie-Centrum-Charité \\ Department of Dermatology and Allergy \\ Charité-Universitätsmedizin Berlin \\ Charitéplatz 1 \\ D-10117 Berlin, Germany \\ E-mail: pavel.kolkhir@charite.de
}

\section{Doi}

10.23822/EurAnnACI.1764-1489.153

To the Editor,

Chronic urticaria (CU) is characterized by wheals and/or angioedema for more than 6 weeks, with a point prevalence of 0.1$1.4 \%(1,2) . \mathrm{CU}$ is classified as chronic spontaneous urticaria $(\mathrm{CSU})$ and chronic inducible urticaria (CIndU), which can occur simultaneously or independently (3). In CSU patients, concomitant CIndU has been associated with severe, long-lasting and/or antihistamine-resistant CSU (4). Omalizumab, an anti-IgE monoclonal antibody, can improve both CSU and CIndU in the same patients $(5,6)$. However, its efficacy in CSU patients with several subtypes of CIndU is poorly characterized $(6,7)$. Here, we describe six patients with antihistamine-resistant CSU and multiple CIndUs (table I) treated with $300 \mathrm{mg}$ omalizumab monthly and followed up for a period of 3-11 months.
Common blood count and serum levels of total IgE, C-reactive protein (CRP), eosinophil cationic protein (ECP), D-dimer and fibrinogen were measured at baseline and 30 days after the injection of omalizumab. The Urticaria Activity Score (UAS) was obtained for 30 days (before and during the treatment). Dermatology Life Quality Index (DLQI) was used at baseline and every 7 days. The Chronic Urticaria Quality of Life Questionnaire (CU-Q2oL) and the Urticaria Control Test (UCT) were applied at baseline and 30 days after the first omalizumab injection. Provocation tests with appropriate triggers were performed according to the international guideline (1). The age of patients ranged from 26 to 52 years (mean: 43 years, table I). The mean duration of CSU and CIndU was two and three years, respectively. In three cases, CIndU appeared before CSU, and in the other three patients CIndU developed after or at the same time as CSU. Provocation tests were positive before treatment in all 
Table I - Demographic characteristics of patients.

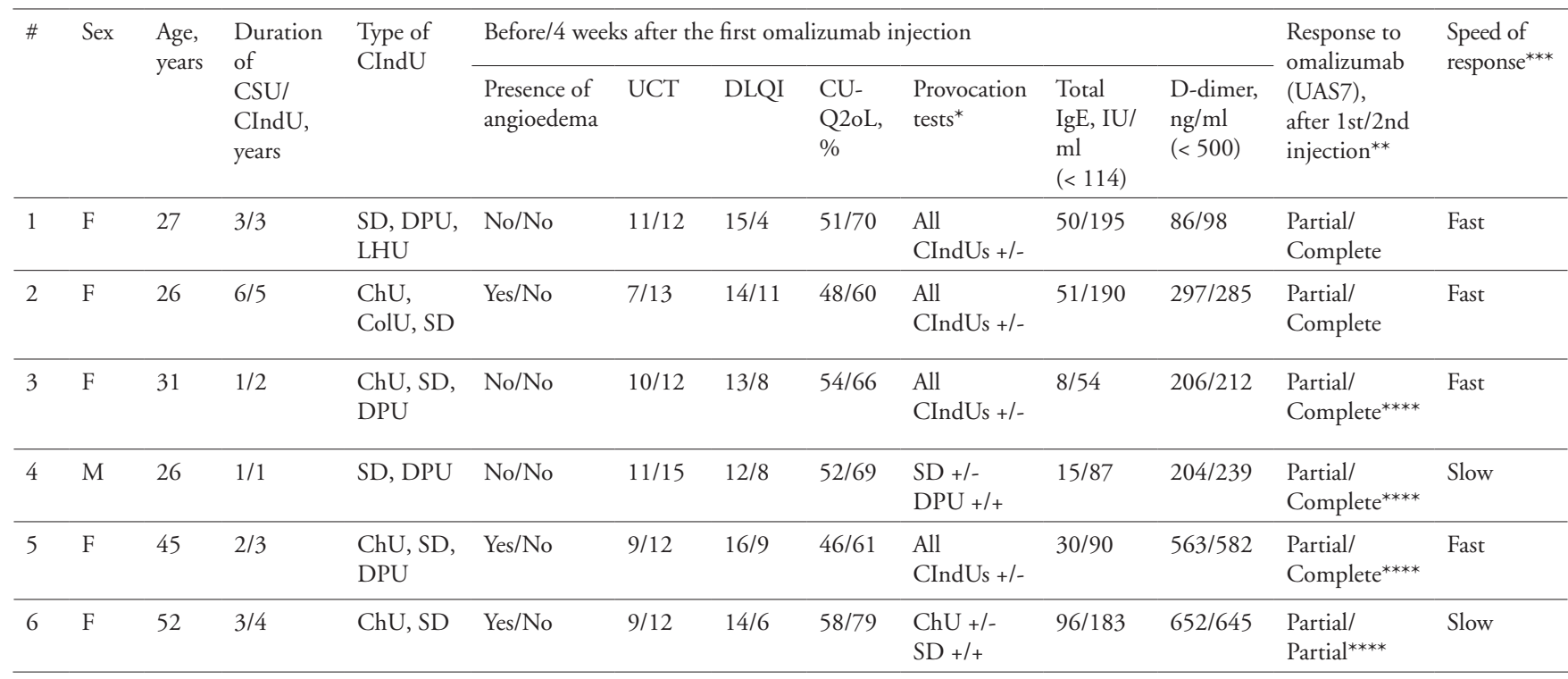

UCT: Urticaria Control Test; DLQI: Dermatology Quality of Life Index; CU-Q2oL: Chronic Urticaria Quality of Life Questionnaire; CIndU: chronic inducible urticaria; F: female; M: male; SD: symptomatic dermographism; DPU: delayed pressure urticaria; LHU: local heat urticaria; ChU: cholinergic urticaria; ColU: cold urticaria; *results of provocation tests: "+" is positive provocation test and "- " is negative provocation test; patients \#4 and \#6 had slightly positive provocation tests for DPU and SD, respectively, after omalizumab treatment; ${ }^{* *}$ complete response: $>90 \%$ reduction from baseline in UAS7 score and partial imrovement: 30 $89 \%$ reduction from baseline in UAS7 score; ${ }^{* * *}$ fast responder: CSU symptoms regressed within 8 days and slow responder: CSU symptoms regressed after 8 days; ${ }^{* * * *}$ response after the second injection was determined by physician global assessment based on patient feedback.

patients. Concomitant symptomatic dermographism (SD), delayed pressure urticaria (DPU) and cholinergic urticaria were diagnosed in six, four, and four patients, respectively. Two and four CSU patients had two and three different subtypes of CIndU, respectively. All patients had wheals and three of them experienced several episodes of angioedema. All patients had uncontrolled disease.

In all patients, disease control was reached, and quality of life was improved within one month after the first omalizumab injection (table I). Provocation tests became negative in four patients. In two patients with SD or DPU, provocation tests remained slightly positive, but everyday CIndU symptoms were gone.

Partial improvement assessed by UAS7 was seen in all patients after the first injection and five of six patients reported complete remission of their CSU after the second injection. Four patients were fast responders and two were slow responders as assessed by UAS and UCT scores (table I). In five patients, the symptoms of CSU and CIndU decreased at the same time. In two slow responders, CIndU symptoms disappeared 2-3 weeks before improvement of CSU symptoms (figure $\mathbf{1} \mathbf{a}-\mathbf{d}$ ).

In all patients, total IgE levels were elevated after the treatment as compared with their baseline values (table I) as described before (8). In the literature, low levels of total $\mathrm{IgE}$ at the baseline have been reported to be associated with nonresponse and/or slow response to omalizumab (9). However, we did not observe this in our patients probably because of the small number of patients included.

Similarly, decrease in CRP and D-dimer levels after successful treatment with omalizumab has been reported (8). In our patients, no difference was seen in levels of ECP, D-dimer, CRP and fibrinogen before and after treatment. In two patients, elevated $\mathrm{D}$-dimer levels were present before the onset of their urticaria and might be associated with concomitant diseases.

The pathomechanism of chronic urticaria is yet to be clearly defined. It is still unknown what causes this activation and degranulation of tissue-resident mast cells and the subsequent release of inflammatory mediators. Type I autoimmunity ("autoallergy") is thought to be a cause of both CIndU and CSU in a subpopulation of patients. Autoallergic urticaria is characterized by the synthesis of autoantigen (autoallergen), which is detected by specific $\mathrm{IgE}$ autoantibodies bound to skin mast cells that resuts in degranulation of mast cells. For example, some IgE autoantibodies have been described in CSU, namely $\mathrm{IgE}$ against thyroid peroxidase, interleukin- 24 and tissue factor. In patients with Type I urticaria, omalizumab can prevent binding of $\operatorname{IgE}$ to the high-affinity $\operatorname{IgE}$ receptor and, therefore, suppress mast cell activation and release of histamine and other mediators. However, some CSU patients respond more slowly to omalizumab that is consistent with Type IIb autoimmunity associated with IgG autoantibodies against IgE and Fc\&RI. In these patients, treatment 
Figure 1 - Patient \#4. CSU: before (a) and after (b) omalizumab treatment. Symptomatic dermographism (results of provocation test with FricTest ${ }^{\circledR}$ device): before (c) and after (d) omalizumab treatment.

(a)

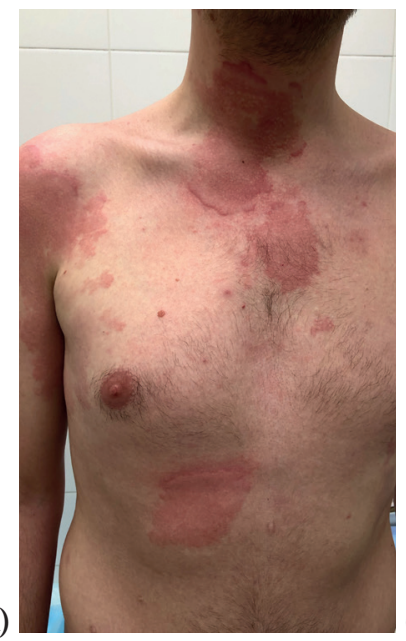

(b)

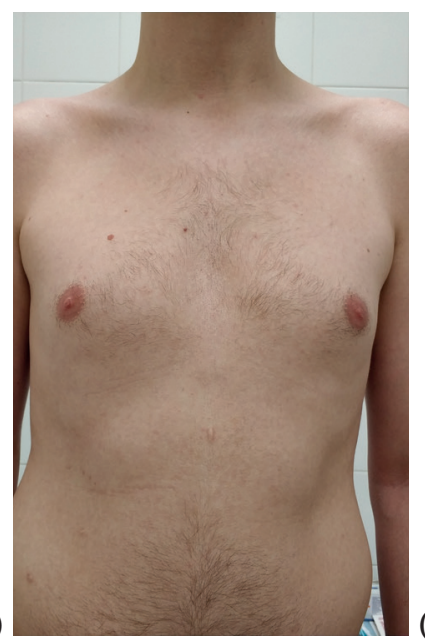

(c)

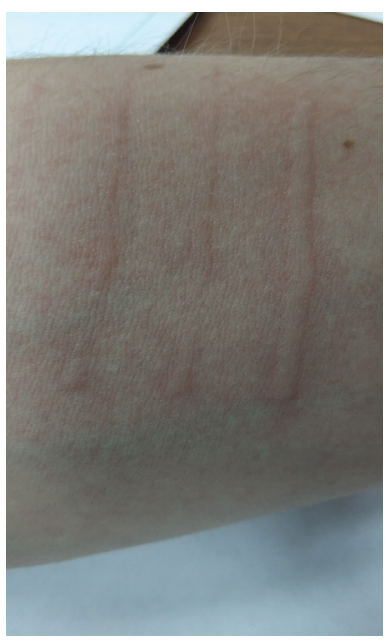

(d)

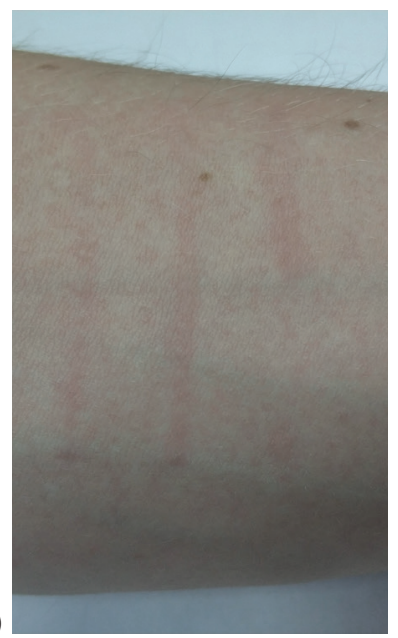

with omalizumab can result in the loss of membrane-bound $\operatorname{IgE}$ and subsequently FceRI from skin mast cells that prevents the activation of mast cells by $\mathrm{IgG}$ autoantibodies $(1,5,10,11)$. In line with other publications $(5,6,10-12)$, our patients with CSU plus several subtypes of CIndU responded well to omalizumab treatment, which resulted in decreased urticaria activity, provocation test responses and increased quality of life and disease control. Prospective treatment studies with omalizumab, in patients with CIndU with and without CSU, children and adults, should be performed.

\section{Acknowledgements}

The study was supported by the "Russian Academic Excellence Project 5-100". Pavel Kolkhir was supported by a GA²LEN fellowship.

\section{Conflict of interests}

Pavel Kolkhir is a speaker for Novartis and Roche. Anastasiia Allenova is a speaker for Novartis.

Dayana Skander has no conflict of interest. Marcus Maurer: grants and/or personal fees from Allakos, Aralez, Alnylam, AstraZeneca, BioCryst, Blueprint, CSL Behring, FAES, Genentech, Kalvista, Menarini, Leo Pharma, Moxie, MSD, Pharming, Pharvaris, Roche, Sanofi, Shire/Takeda, UCB, Uriach, outside the submitted work.

\section{References}

1. Zuberbier T, Aberer W, Asero R, et al. The EAACI/GA(2)LEN/ $\mathrm{EDF} / \mathrm{WAO}$ guideline for the definition, classification, diagnosis and management of urticaria. Allergy 2018;73(7):1393-414.

2. Fricke J, Ávila G, Keller T, et al. Prevalence of chronic urticaria in children and adults across the globe: Systematic review with meta-analysis. Allergy 2019;75(2):423-432.
3. Sanchez J, Amaya E, Acevedo A, Celis A, Caraballo D, Cardona R. Prevalence of Inducible Urticaria in Patients with Chronic Spontaneous Urticaria: Associated Risk Factors. J Allergy Clin Immunol Pract 2017;5(2):464-70.

4. Sanchez-Borges M, Caballero-Fonseca F, Capriles-Hulett A, Gonzalez-Aveledo L, Maurer M. Factors linked to disease severity and time to remission in patients with chronic spontaneous urticaria. J Eur Acad Dermatol Venereol 2017;31(6):964-71.

5. Maurer M, Metz M, Brehler R, et al. Omalizumab treatment in patients with chronic inducible urticaria: A systematic review of published evidence. Journal Allergy Clin Immunol 2018;141(2):638-49.

6. Vieira Dos Santos R, Locks Bidese B, Rabello de Souza J, Maurer M. Effects of omalizumab in a patient with three types of chronic urticaria. Br J Dermatol 2014;170(2):469-71.

7. Marcelino J, Costa AC, Mendes A, et al. Omalizumab in chronic spontaneous and inducible urticaria: a 9 year retrospective study in Portugal. Eur Ann Allergy Clin Immunol 2018;50(4):169-76.

8. de Montjoye L, Darrigade A-S, Giménez-Arnau A, Herman A, Dumoutier L, Baeck M. Correlations between disease activity, autoimmunity and biological parameters in patients with chronic spontaneous urticaria. Eur Ann Allergy Clin Immunol 2020;53(2):55-66.

9. Ertas R, Ozyurt K, Atasoy M, Hawro T, Maurer M. The clinical response to omalizumab in chronic spontaneous urticaria patients is linked to and predicted by $\operatorname{IgE}$ levels and their change. Allergy 2018;73(3):705-12.

10. Metz M, Ohanyan T, Church MK, Maurer M. Omalizum$\mathrm{ab}$ is an effective and rapidly acting therapy in difficult-to-treat chronic urticaria: a retrospective clinical analysis. J Dermatol Sci 2014;73(1):57-62.

11. Metz M, Ohanyan T, Church MK, Maurer M. Retreatment with omalizumab results in rapid remission in chronic spontaneous and inducible urticaria. JAMA Dermatol 2014;150(3):288-90.

12. Kocaturk E, Can PK, Akbas PE, et al. Management of chronic inducible urticaria according to the guidelines: A prospective controlled study. J Dermatol Sci 2017;87(1):60-9. 